\title{
LOCAL AND GLOBAL INTERPOLATION INEQUALITIES ON THE FOLLAND-STEIN SOBOLEV SPACES AND POLYNOMIALS ON STRATIFIED GROUPS
}

\author{
GuOzhen Lu
}

\begin{abstract}
We derive both local and global Sobolev interpolation inequalities of any higher orders for the Folland-Stein Sobolev spaces on stratified nilpotent Lie groups $\mathbb{G}$ and on domains satisfying a certain chain condition. Weighted versions of such inequalities are also included for doubling weights satisfying a weighted Poincaré inequality. This paper appears to be the first one to deal with general Sobolev interpolation inequalities for vector fields on Lie groups; Despite the extensive research for Poincaré type inequalities for vector fields over the years, interpolation inequalities given here even in the nonweighted case appear to be new. Such interpolation inequalities have important applications to subelliptic or parabolic pde's involving vector fields. The main tools to prove such inequalities are approximating the functions by polynomials on $\mathbb{G}$. Some very useful properties for projections of polynomials associated with the functions are given here and they appear to have independent interests in their own rights. Main ideas of the proofs of the theorems are explained here. Detailed proofs of the results presented here are given in the paper "Polynomials on stratified groups and Sobolev interpolation inequalities on nonisotropic Folland-Stein spaces" by the author.
\end{abstract}

\section{Introduction}

(Weighted) Sobolev interpolation inequalities have been extensively studied in the classic case, see Chua [C], Gutierrez-Wheeden [GW1], Caffarelli-KohnNirenberg [CKN1], Lin [Lin] and many references therein. These inequalities have proved to be rather powerful in studying various aspects of partial differential equations, especially for pde's of elliptic and parabolic type. In particular, such inequalities are the main tool in proving Harnack's inequality for solutions of certain degenerate parabolic equations by adapting Moser's argument [M] (see Gutierrez-Wheeden [GW2-3]). Another application of weighted Sobolev interpolation inequalities can be found in the work of Caffarelli-Kohn-Nirenberg [CKN2], where they applied the results in [CKN1] to study the partial regularity of weak solutions to Navier-Stokes equations. However, such inequalities, even in the nonweighted case which is often of equal importance and general interests, have not been established on stratified groups, or more generally for degenerate

Received June 20, 1997.

1991 Mathematics Subject Classification. Primary 26D10, 46E35, 22E25, 35K65.

Key words and phrases. Poincaré inequalities, doubling weights, stratified and nilpotent Lie groups, vector fields, $A_{p}$ weights, polynomials on Lie groups, Sobolev interpolation inequalities.

Partially supported by the National Science Foundation Grant \#DMS96-22996. 
vector fields. In this paper, we will study both nonweighted and weighted, local and global Sobolev interpolation inequalities. We shall state these different versions separately for the purpose of clarity. For a reader who is only interested in the nonweighted results, we refer him to theorems (3.2), (3.3), (3.4) in Section 3 and theorems (4.1) and (4.2) in Section 4.

For weighted inequalities, we are concerned with weights $w, v$ together with $1 \leq p \leq q<\infty$ satisfying the following inequality:

$$
\left\|f-f_{B}\right\|_{L_{w}^{q}(B)} \leq A(B)\|X f\|_{L_{v}^{p}(B)}
$$

where $B \subset \mathbb{G}$ is a metric ball, $f_{B}=\int_{B} f d x /|B|,|X f|=\left(\sum_{i=1}^{m}\left|X_{i} f\right|^{2}\right)^{1 / 2}$ and $w(E)=\int_{E} w(x) d x$. Let us note that some sufficient and necessary conditions have been obtained for (1.1) for Grushin and Hormander vector fields; see [FGW] and $[\mathrm{FLW}$. For instance, when $v$ and $w$ satisfy a certain balance condidtion first introduced in Chanillo-Wheeden $[\mathrm{CW}]$ and $v \in A_{p}$, then (1.1) holds. In particular, in this case $A(B)$ can be taken as $C w(B)^{1 / q} v(B)^{-1 / p} \rho(B)$.

To state our theorems, we need to introduce briefly some notions about groups. Let $\mathcal{G}$ be a finite-dimensional, stratified, nilpotent Lie algebra. Assume

$$
\mathcal{G}=\oplus_{i=1}^{s} V_{i},
$$

and $\left[V_{i}, V_{j}\right] \subset V_{i+j}$ for $i+j \leq s,\left[V_{i}, V_{j}\right]=0$ for $i+j>s$. Let $X_{1}, \ldots, X_{m}$ be a basis for $V_{1}$ and suppose that $X_{1}, \ldots, X_{m}$ generate $\mathcal{G}$ as a Lie algebra. Thus we can choose a basis $\left\{X_{i j}\right\}$, for $1 \leq j \leq s, 1 \leq i \leq m_{j}$ for $V_{j}$ consisting of vectors of the form $X_{\alpha}$ for some multi-indices $\alpha$ of length $j$. In particular, $X_{i 1}=X_{i}, i=1, \ldots, m$ and $m=m_{1}$.

Let $\mathbb{G}$ be the simply connected Lie group associated to $\mathcal{G}$. Since the exponential mapping is a global diffeomorphism from $\mathcal{G}$ to $\mathbb{G}$, for each $g \in \mathbb{G}$, there is $x=\left(x_{i j}\right) \in \mathbb{R}^{N}, 1 \leq i \leq m_{j}, 1 \leq j \leq s, N=\sum_{j=1}^{s} m_{j}$, such that

$$
g=\exp \left(\sum x_{i j} X_{i j}\right) .
$$

Thus we define a homogeneous norm function $|\cdot|$ on $G$ by

$$
|g|=\left(\sum\left|x_{i j}\right|^{2 s ! / j}\right)^{1 / 2 s !} .
$$

We also define $Q=\sum_{j=1}^{s} j m_{j}$, which is called the homogeneous dimension of $\mathbb{G}$, which is usually greater than $\operatorname{dim} \mathbb{G}=N$.

Now let $\varrho: \mathbb{G} \times \mathbb{G} \rightarrow \mathbb{R}^{+}$be defined by $\varrho(x, y)=\left|x y^{-1}\right|$, the homogeneous norm of $x y^{-1}$. We denote by $B(x, r)=\{y \in \mathbb{G}: \varrho(x, y)<r\}$, the ball centered at $x$ and with radius $r$. We note that $(\mathbb{G}, \varrho)$ is a homogeneous metric space in the sense of Coifman and Weiss $([\mathrm{CoW}])$. Thus, weighted theory in such spaces can be developed as in [Ca].

We now define the so-called Boman chain domain in $\mathbb{G}$.

Definition 1.1. An open set $\Omega$ in $\mathbb{G}$ is said to be a member of $\mathcal{F}(\sigma, N), \sigma \geq 1$, $N \geq 1$, if there exists a covering $W$ of $\Omega$ consisting of balls such that: 


$$
\sum_{B \in W} \chi_{\sigma B}(x) \leq N \chi_{\Omega}(x) \quad \forall x \in \mathbb{G} .
$$

(ii) There is a 'central ball' $B_{0} \in W$ that can be connected with every ball $B \in W$ by a finite chain of balls $B_{0}, B_{1}, \cdots, B_{k(B)}=B$ from $W$ such that $B \subset N B_{j}$ for $j=0,1, \cdots, k(B)$. Moreover, $B_{j} \cap B_{j+1}$ contains a ball $R_{j}$ such that $B_{j} \cup B_{j+1} \subset N R_{j}$.

We say that $\Omega$ satisfies the Boman chain condition [B] if $\Omega \in \mathcal{F}(\sigma, N)$ for some $N, \sigma \geq 1$. There are many types of domains satisfy the Boman chain condition, for examples, metric balls (see [FGW], [L2]), and John domains (see [BKL] and $[\mathrm{GN}])$.

In what follows, $B$ is always a ball and $\rho(B)$ will be its radius. If $1<p<\infty$, $p^{\prime}$ will denote $p /(p-1)$. By a weight $w$, we mean a non-negative locally integrable function on $\mathbb{G}$. By abusing notation, we will also write $w$ for the measure induced by $w$. Sometimes we write $d w$ to denote $w d x$. We say that $w$ is doubling if $w(2 B) \leq C w(B)$ for every ball $B$, where $2 B$ denotes the ball with the same center as $B$ and twice its radius. By $w \in A_{p}$, we mean $w$ satisfies the Muckenhoupt $A_{p}$ condition, i.e.,

$$
\begin{gathered}
\frac{1}{|B|}\left(\int_{B} w d x\right)^{1 / p}\left(\int_{B} w^{\frac{-1}{p-1}} d x\right)^{1 / p^{\prime}} \leq C \text { when } 1<p<\infty, \text { and } \\
\frac{1}{|B|} \int_{B} w(x) d x \leq C \operatorname{essinf}_{x \in B} w(x) \text { when } p=1,
\end{gathered}
$$

for all balls $B$ in $\mathbb{G}$. Note that $w$ is doubling when it is in $A_{p}$.

Let $\Omega$ be an open set in $\mathbb{G}$. If $\alpha$ is a multi-index, $\alpha=\left(\alpha_{1}, \alpha_{2}, \ldots, \alpha_{l}\right) \in \mathbb{Z}_{+}^{l}$, we will denote $\sum_{j=1}^{l} \alpha_{j}$ by $|\alpha|$ and $X^{\alpha}=X_{i_{1}}^{\alpha_{1}} \cdots X_{i_{l}}^{\alpha_{l}}, 1 \leq i_{j} \leq m$. We denote by $X$ the vector $\left(X_{1}, \cdots, X_{m}\right)$ and by $X^{k}$ the vector of all possible $k^{\text {th }}$ order derivatives $\left\{X^{\alpha}\right\}_{|\alpha|=k}$ for $k \in \mathbb{N}$. A locally integrable function $f$ on $\Omega$ (we will write $\left.f \in L_{\text {loc }}^{1}(\Omega)\right)$ has a weak derivative of order $\alpha$ if there is a locally integrable function (denoted by $X^{\alpha} f=X_{i_{1}}^{\alpha_{1}} \cdots X_{i_{l}}^{\alpha_{l}} f$ ) such that

$$
\int_{\Omega} f\left(X_{i_{1}}^{\alpha_{1}} \cdots X_{i_{l}}^{\alpha_{l}} \varphi\right) d x=(-1)^{|\alpha|} \int_{\Omega}\left(X_{i_{l}}^{\alpha_{l}} \cdots X_{i_{1}}^{\alpha_{1}} f\right) \varphi d x
$$

for all $C^{\infty}$ functions $\varphi$ with compact support in $\Omega$, i.e., $\varphi \in C_{0}^{\infty}(\Omega)$.

For $1 \leq p<\infty, k \in \mathbb{N}$, and any weight $w, L_{w, k}^{p}(\Omega)$ and $E_{w, k}^{p}(\Omega)$ are the weighted Folland-Stein Sobolev spaces of functions having weak derivatives of all orders $\alpha,|\alpha| \leq k$, and satisfying

$$
\|f\|_{L_{w, k}^{p}(\Omega)}=\sum_{0 \leq|\alpha| \leq k}\left\|X^{a} f\right\|_{L_{w}^{p}(\Omega)}=\sum_{0 \leq|\alpha| \leq k}\left(\int_{\Omega}\left|X^{\alpha} f\right|^{p} d w\right)^{1 / p}<\infty,
$$

and

$$
\|f\|_{E_{w, k}^{p}(\Omega)}=\sum_{|\alpha|=k}\left\|X^{\alpha} f\right\|_{L_{w}^{p}(\Omega)}<\infty
$$


respectively. Moreover, in the case when $w \equiv 1$, we will denote $L_{w, k}^{p}(\Omega)$ and $E_{w, k}^{p}(\Omega)$ by $L_{k}^{p}(\Omega)$ and $E_{k}^{p}(\Omega)$ respectively. Finally, let $\Lambda^{k}(\Omega)$ be the collection of all functions $f$ on $\Omega$ such that all its weak derivatives $X^{\alpha}$ of order $|\alpha| \leq k$ exist.

The organization of the paper is as follows: In section 2, we give several important properties regarding polynomials on $\mathbb{G}$, especially the comparison, projection and approximation theorems. These remarkable properties of group polynomials appear to be very useful in proving such interpolation inequalities. Moreover, these properties are as well of independent interests in their own rights and other applications will be found in the forthcoming works. In particular we will show the projection polynomials $\pi_{k}(B) f$ associated to $B$ and $f$ satisfying

$$
\left\|X^{I} \pi_{k}(B) f\right\|_{L_{w}^{q}(B)} \leq C\left\|X^{l} f\right\|_{L_{w}^{q}(B)}
$$

for $1 \leq q \leq \infty, 0 \leq|I|=l<\infty$ and $w \in A_{q}$ when $q \neq \infty$. Section 3 gives interpolation inequalities of zero and lower orders which are particularly important to study pde's of second order. Section 4 deals with inequalities of any higher orders. We also give some Poincaré type estimates for derivatives of higher orders in Section 5.

\section{Polynomials on $\mathbb{G}$ and their important properties}

We now define polynomials on $\mathbb{G}$ by following Folland-Stein (see [FS2]). Let $X_{1}, \cdots, X_{m}$ be the generators of the Lie algebra $\mathcal{G}$, and $X_{1}, \cdots, X_{m}, \cdots, X_{N}$ be a basis of $\mathcal{G}$. We denote $d\left(X_{j}\right)=d_{j}$ as the length of $X_{j}$ as a commutator, and $1 \leq d_{1} \leq \cdots \leq d_{N}$. Thus it is easy to see $d_{j}=1$ for $j=1, \cdots, m$. Let $\xi_{1}, \cdots, \xi_{N}$ be the dual basis for $\mathcal{G}^{*}$, and let $\eta_{k}=\xi_{k} \cdot \exp ^{-1}$. Thus $\eta_{1}, \cdots, \eta_{N}$ are a system of global coordinates on $\mathbb{G}$. A function $P$ on $\mathbb{G}$ is called a polynomial on $\mathbb{G}$ if $P \cdot \exp$ is a polynomial on $\mathcal{G}$. By this definition, $\eta_{1}, \cdots, \eta_{N}$ are polynomials on $\mathbb{G}$ and generate the algebra of polynomials on $\mathbb{G}$. Thus every polynomial on $\mathbb{G}$ can be written uniquely as

$$
P=\sum_{I} a_{I} \eta^{I},\left(\eta^{I}=\eta_{1}^{i^{1}} \cdots \eta_{N}^{i_{N}}, a_{I} \in C\right),
$$

where all but finitely many of coefficients $a_{I}$ vanish. Clearly $\eta^{I}$ is homogeneous of degree $d(I)$, where $d(I)=\sum_{j=1}^{N} i_{j} d\left(i_{j}\right)$. If $P=\sum_{I} a_{I} \eta^{I}$, then we define its homogeneous degree as $\max \left\{d(I): a_{I} \neq 0\right\}$ and $\max \left\{|I|: a_{I} \neq 0\right\}$ as its isotropic degree. If we consider $I=\left(i_{1}, \cdots, i_{m}\right), 1 \leq i_{j} \leq m$, then $d(I)=|I|$.

Polynomials on homogeneous groups bear some resemblence to those in the Euclidean spaces. We refer the reader to Folland-Stein [FS2] for more details.

In what follows, $C$ denotes various positive constants. They may differ even in a same string of estimates. Moreover, sometimes, we will use $C(\alpha, \beta, \cdots)$ instead of $C$ to emphasize that the constant is depending on $\alpha, \beta, \cdots$. Throughout this section and the rest of the paper, $w$ is always denoted a doubling weight on $\mathbb{G}$. 
When $w=1$, all the theorems below still hold with the measures $w(E)$ replaced by the Lebesgue measure $|E|$.

We can obtain the following theorems:

Theorem 2.1 (Comparison). Let $\gamma>0$ be a constant. Let $F, B$ be balls in $\mathbb{G}$ such that $F \subset B$ and $|F|>\gamma|B|$. If $w$ is a doubling weight, $1 \leq q<\infty$, and $P$ is a polynomial of degree $k$, then

$$
\|P\|_{L_{w}^{q}(E)} \leq C(\gamma, k, n, w)\left(\frac{w(E)}{w(F)}\right)^{1 / q}\|P\|_{L_{w}^{q}(F)}
$$

for all measurable sets $E \subset B$.

Given $k$ and a polynomial $P$ of degree $k$,

$$
\|P\|_{L^{\infty}(B)} \leq \frac{C}{w(B)}\|P\|_{L_{w}^{1}(B)}
$$

with $C$ independent of $B$ and $P$. This can be seen through the equivalence of two norms on the finite dimensional space.

Lemma 2.2. Let $B$ be a ball and let $E$ be a measurable set in $B$ with $|E|>\gamma \mid$ $B \mid$. If $P$ is a polynomial of degree $k$, then

$$
\|P\|_{L^{\infty}(E)} \geq C(\gamma, k)\|P\|_{L^{\infty}(B)} .
$$

We also can derive the Bernstein's inequality:

Theorem 2.3. Let $P$ be a polynomial of order less than $k$ and $1 \leq q \leq \infty$. Let $I$ be any multi-index and $w$ be doubling, then

$$
\left\|X^{I} P\right\|_{L_{w}^{q}(B)} \leq C \rho(B)^{-|I|}\|P\|_{L_{w}^{q}(B)} \text { for all balls } B \text { in } \mathbb{G}
$$

where $C$ depends only on $k, w, q$.

Let $\mathcal{P}_{k}$ be the collection of all polynomials of degree $<k$ on $\mathbb{G}$. Then we have the following theorem concerning the projection of function into polynomials. This projection theorem is crucial to the proof of Sobolev interpolation inequalities and many other purposes given elsewhere.

Theorem 2.4 (Projection). For each $k \in \mathbb{N}$ and balls $B \subset \Omega$, there exists a projection $\pi_{k}(B): \Lambda^{k}(\Omega) \longrightarrow \mathcal{P}_{k}$ such that

$$
\operatorname{esssup}_{x \in B}\left|\pi_{k}(B) f(x)\right| \leq C \rho(B)^{-Q}\|f\|_{L^{1}(B)}
$$

with $C$ independent of $f$ and $B$. Moreover, $\pi_{k}(B)$ is linear and $\pi_{k}(B) P=P$ for all $P \in \mathcal{P}_{k}$, and for all $1 \leq q \leq \infty$ and a doubling weight $w$,

$$
\left\|\pi_{k}(B) f\right\|_{L_{w}^{q}(B)} \leq C\|f\|_{L_{w}^{q}(B)} .
$$


What is even more interesting is the following property of the above projection polynomials. The following theorem (2.6) given below states that not only can the projection polynomial of a function, but also its subelliptic derivatives be controlled by the subelliptic derivatives of the function in any $L^{q}$ norms. This theorem can not be proved by simply using change of variables, even we know the corresponding inequalities are true in the Euclidean spaces. This is due to the fact that there is no cannonical change of variables mapping the subelliptic vector fields on the group $\mathbb{G}$ into the partial derivatives in $\mathbb{R}^{N}$. However, we can derive this by using the approximation polynomials, together with some simple but delicate argument.

We first give a theorem which is, among many other applications, also very useful for the proof of Sobolev interpolation inequalities by approximating functions by polynomials on each metric ball.

Theorem 2.5 (Approximation). Let $\Omega \subset \mathbb{G}$ be an open set. Then given any positive integer $k$ and $f \in \Lambda^{k}(\mathbb{G})$, there exists a polynomial $P=P(f, \Omega)$ on $\mathbb{G}$ of degree less than $k$ such that

$$
\int_{\Omega} X^{\alpha}(f-P)=0, \text { for any } 0 \leq|\alpha|<k,
$$

where $X^{\alpha}=X_{i_{1}}^{\alpha_{1}} \cdots X_{i_{m}}^{\alpha_{m}}$ and $1 \leq i_{j} \leq m$.

The proof is given by examining closely the definition of polynomials on the stratified groups. We first observe that for each multi-index $I$ and $J$ with the same length $d(I)=d(J)$ we have

$$
X^{I}\left(\eta^{J}\right)=I ! \delta_{I J}, \text { where } \delta_{I J}=1 \text { if } I=J ; \text { and }=0 \text { if } I \neq J .
$$

This follows from the definition of the dual basis $\eta_{1}, \cdots, \eta_{N}$ on $\mathcal{G}^{*}$, which indicates the inner product defining the dual basis satisfies

$$
<X^{J}, \xi^{I} \circ \exp ^{-1}>=I ! \delta_{I J} .
$$

We also note that for any polynomial $P$ of degree less than $k$ we have $X^{\alpha} P=0$ for any $|\alpha|>k$.

Since any polynomial $P$ on $\mathbb{G}$ of degree less than $k$ can be uniquely written as

$$
P=\sum_{I: d(I)<k} a_{I} \eta^{I}, \eta^{I}=\eta_{j_{1}}^{i_{1}} \cdots \eta_{j_{N}}^{i_{N}}
$$

we thus only need to determine the coefficient $a_{I}$.

If $X^{J}(P)=a_{J} J$ ! were true, and $\int_{\Omega} X^{J} f=\int_{\Omega} X^{J} P$ for each $J$ satisfying $d(J)<k$, thus one would be attempted to take

$$
a_{J}=\frac{1}{J !|\Omega|} \int_{\Omega} X^{J} f
$$


However, we note (2.2) is true only for $d(I)=d(J)$ and not necessarily true for $d(I) \neq d(J)$, especially for $I \subset J$ (i.e., all the indices appeared in $I$ appear in $J$ as well). Thus, $X^{J}(P)=a_{J} J$ ! is not necessarily true.

Fortunately, this can be overcome by an iteration procedure. Details are given in [L3].

By using the same projection given in Theorem (2.4), we can get with the aid of Theorem (2.5) the following important

Theorem 2.6. For each $k \in \mathbb{N}$ and balls $B \subset \Omega$, there exists a projection $\pi_{k}(B): \Lambda^{k}(\Omega) \longrightarrow \mathcal{P}_{k}$ such that

$$
\operatorname{esssup}_{x \in B}\left|\pi_{k}(B) f(x)\right| \leq C \rho(B)^{-Q}\|f\|_{L^{1}(B)}
$$

and for any $1 \leq q \leq \infty, w \in A_{q}$ for $q \neq \infty$ and any multiple index $I$ with $|I|=l \geq 0$

$$
\left\|X^{I} \pi_{k}(B) f\right\|_{L_{w}^{q}(B)} \leq C\left\|X^{l} f\right\|_{L_{w}^{q}(B)}
$$

with $C$ independent of $f$ and $B$. Moreover, $\pi_{k}(B)$ is linear and $\pi_{k}(B) P=P$ for all $P \in \mathcal{P}_{k}$.

We remark here that the projection polynomials constructed in Theorems (2.4) and (2.6) are in general not the same as those in Theorem (2.5). Each of them has its own merit and different one is often taken for different purposes.

\section{Interpolation inequalities of lower orders}

We will state in this section Sobolev interpolation inequalities of zero and first orders and results involved with Schrödinger operator $-\sum_{i=1}^{m} X_{i}(x)+V(x)$ when $V$ is a nonnegative polynomial on $\mathbb{G}$. These inequalities are particularly useful to study subelliptic or parabolic equations of second order, for instance, subelliptic or parabolic Schrodinger operators $\frac{\partial}{\partial t}=-\sum_{i=1}^{m} X_{i}^{2}+V$. We recall that $Q$ is the homogeneous dimension of $\mathbb{G}$. We remark once for all that all the local theorems (thus global ones) can be stated by replacing the $L^{1}$ norms by $L^{r}$ norms for $r>1$ in view of the Hölder's inequality. But we will not do this in all the cases.

Let $1 \leq p \leq q<\infty$ satisfy

$$
\left(\frac{1}{|B|} \int_{B}\left|f-f_{B}\right|^{q}\right)^{\frac{1}{q}} \leq C \rho(B)\left(\frac{1}{|B|} \int_{B}|X f|^{p}\right)^{\frac{1}{p}} .
$$

Such an inequality is known to hold for suitable $p, q$, for example, $\frac{1}{p}-\frac{1}{q} \leq \frac{1}{Q}$ for $1 \leq p<Q$ and $p=Q, 1 \leq q<\infty$ (see for example for $p>1$ in [L2], and for $p=1$ in [FLW] $)$. Then we have the following nonweighted Sobolev interpolation inequalities. 
Theorem 3.1 (Global). Let $1 \leq p \leq q<\infty$ such that (3.1) holds for all $B \in \mathbb{G}$ and $f \in \Lambda^{1}(\mathbb{G})$, then for any $1 \leq r \leq q$ we have

$$
\|f\|_{L^{q}(\mathbb{G})} \leq\|f\|_{L^{r}(\mathbb{G})}^{\frac{1+Q\left(\frac{1}{q}-\frac{1}{p}\right)}{1+Q\left(\frac{1}{p}-\frac{1}{p}\right)}} \cdot\|X f\|_{L^{p}(\mathbb{G})}^{\frac{Q\left(\frac{1}{r}-\frac{1}{q}\right)}{1+Q\left(\frac{1}{r}-\frac{1}{p}\right)}}
$$

for all $f \in \Lambda^{1}(\mathbb{G})$ provided that $\|X f\|_{L^{p}(\mathbb{G})} \neq 0$.

Theorem 3.2 (Local). Let $1 \leq p \leq q<\infty$ satisfy (3.1) and $f \in \Lambda^{1}(\bar{B})$, then

$$
\begin{aligned}
\|X f\|_{L^{q}(B)} \leq & C|B|^{1 / q} \rho(B)^{-Q}\left(\rho(B)^{-1}\|f\|_{L^{1}(B)}+\rho(B)\left\|X^{2} f\right\|_{L^{1}(B)}\right) \\
& +C|B|^{\frac{1}{Q}-\frac{1}{q}+\frac{1}{p}}\left\|X^{2} f\right\|_{L^{p}(B)}
\end{aligned}
$$

for all $f \in \Lambda^{2}(B)$, where $C$ is independent of $f$ and $B$.

Theorem 3.3 (Global). Let $1 \leq p \leq q<\infty$ satisfy (3.1) for all balls $B \subset \mathbb{G}$, $1 \leq r \leq q$ and $f \in \Lambda^{1}(\mathbb{G})$, then

$$
\|X f\|_{L^{q}(\mathbb{G})} \leq\|f\|_{L^{r}(\mathbb{G})}^{\frac{\frac{Q}{q}-\frac{Q}{p}+1}{2+\frac{Q}{p}-\frac{Q}{p}}} \cdot\left\|X^{2} f\right\|_{L^{p}(\mathbb{G})}^{\frac{1+\frac{Q}{r}-\frac{Q}{q}}{2+\frac{Q}{p}}}
$$

for all $f \in \Lambda^{2}(\mathbb{G})$ provided that $\left\|X^{2} f\right\|_{L^{p}(\mathbb{G})} \neq 0$.

By combining Theorem (3.3) with the $L^{p}$ estimates derived in [L4] and [L5] we get the following theorem concerning the Schrödinger operators:

Theorem 3.4 (Global). Let $1<p \leq q<\infty$ such that (3.1) holds for all $B \in \mathbb{G}$ and $f \in \Lambda^{1}(\mathbb{G})$ and $1 \leq r \leq q$, then for any nonnegative polynomial $V$ on $\mathbb{G}$,

$$
\|X f\|_{L^{q}(\mathbb{G})} \leq\|f\|_{L^{r}(\mathbb{G})}^{\frac{\frac{Q}{q}-\frac{Q}{p}+1}{2+\frac{Q}{p}-\frac{Q}{p}}} \cdot\left\|\left(-\sum_{i=1}^{m} X_{i}^{2}+V\right) f\right\|_{L^{p}(\mathbb{G})}^{\frac{1+\frac{Q}{r}-\frac{Q}{q}}{2+\frac{Q}{p}-\frac{Q}{p}}}
$$

for all $f \in \Lambda^{2}(\mathbb{G})$.

Let $1 \leq p \leq q<\infty$ and $w, v$ be two weights satisfying

$$
\left(\frac{1}{w(B)} \int_{B}\left|f-f_{B}\right|^{q} w\right)^{\frac{1}{q}} \leq C \rho(B)\left(\frac{1}{v(B)} \int_{B}|X f|^{p} v\right)^{\frac{1}{p}} .
$$

Such an inequality is known to be true for suitable $p, q$ and $w, v$ satisfying a certain balance condition and $v \in A_{p}$ (see [FLW] for Hörmander vector fields and $[\mathrm{CW}],[\mathrm{SW}],[\mathrm{FGW}]$ for other situations). Then for weights $w, v$ satisfying (3.2) we have the following 
Theorem 3.5 (local). Let $1 \leq p \leq q<\infty$ and $w, v$ satisfy (3.2) holds for all $B \in \mathbb{G}$ and $f \in \Lambda^{1}(\mathbb{G})$, then we have

$$
\begin{aligned}
\|f\|_{L_{w}^{q}(B)} & \leq\|f\|_{L_{w}^{q}(B)} \\
& \leq C w(B)^{\frac{1}{q}} v(B)^{-\frac{1}{p}} \rho(B)\|X f\|_{L_{v}^{p}(B)}+C w(B)^{\frac{1}{q}}|B|^{-1}\|f\|_{L^{1}(B)}
\end{aligned}
$$

for all $f \in \Lambda^{1}(\bar{B})$.

Theorem 3.6 (Local). Let $1 \leq p \leq q<\infty$. If $v$ is a weight and $w$ is a doubling weight such that (3.2) holds for all $B \in \mathbb{G}$ and $f \in \Lambda^{1}(\bar{B})$, then

$$
\begin{aligned}
\|X f\|_{L_{w}^{q}(B)} & \leq C w(B)^{1 / q} \rho(B)^{-Q}\left(\rho(B)^{-1}\|f\|_{L^{1}(B)}+\rho(B)\left\|X^{2} f\right\|_{L^{1}(B)}\right) \\
& +C w(B)^{1 / q} v(B)^{-1 / p} \rho(B)\left\|X^{2} f\right\|_{L_{v}^{p}(B)}
\end{aligned}
$$

for all $f \in \Lambda^{2}(B)$, where $C$ is independent of $f$ and $B$.

Theorem 3.7 (Chain domain). Let $\Omega \in \mathcal{F}(\sigma, N)$ and let $W$ be a covering of $\Omega$ satisfying the Boman chain condition. Let $1 \leq p \leq q<\infty$. If $v$ is a weight and $w$ is a doubling weight such that (3.2) holds for all $B \in W$ and $f \in \Lambda^{1}(\Omega)$, then

$$
\begin{aligned}
\|X f\|_{L_{w}^{q}(\Omega)} \leq & C w\left(B_{0}\right)^{1 / q} \rho\left(B_{0}\right)^{-Q}\left(\rho\left(B_{0}\right)^{-1}\|f\|_{L^{1}\left(B_{0}\right)}+\rho\left(B_{0}\right)\left\|X^{2} f\right\|_{L^{1}\left(B_{0}\right)}\right) \\
& +C A_{0}\left\|X^{2} f\right\|_{L_{v}^{p}(\Omega)}
\end{aligned}
$$

for all $f \in \Lambda^{2}(\Omega)$ where $A_{0}=\sup _{B \in W} A(B), A(B)=\rho(B) w(B)^{\frac{1}{q}} v(B)^{\frac{-1}{p}}, B_{0}$ is the 'central' ball in $W$, and $C$ is independent of $f$.

\section{Interpolation inequalities of higher orders}

In this section we give some interpolation inequalities of higher orders. As in the same pattern in the last subsection, we shall state the nonweighted case first.

Theorem 4.1 (Local). Let $1 \leq p \leq q<\infty$. Let $i, k \in \mathbb{N}, 1 \leq i<k$. Then

$$
\left\|X^{i} f\right\|_{L^{q}(B)} \leq C|B|^{1 / q} \rho(B)^{-i-Q}\|f\|_{L^{1}(B)}+C|B|^{1 / q-1 / p} \rho(B)^{k-i}\left\|X^{k} f\right\|_{L^{p}(B)}
$$

for all $f \in \Lambda^{k}(B)$ for all balls $B$.

Theorem 4.2 (Global). Let $1 \leq p \leq q<\infty$ such that (3.1) holds. Let $i, k \in$ $\mathbb{N}, 1 \leq i<k$ and $1 \leq r \leq q$. Then

$$
\left\|X^{i} f\right\|_{L^{q}(\mathbb{G})} \leq C\|f\|_{L^{r}(\mathbb{G})}^{\frac{\frac{Q}{q}+k-\frac{Q}{p}-i}{p+\frac{Q}{p}}} \cdot\left\|X^{k} f\right\|_{L^{p}(\mathbb{G})}^{\frac{\frac{Q}{r}+i-\frac{Q}{q}}{k+\frac{Q}{p}}}
$$

for all $f \in \Lambda^{k}(\mathbb{G})$ provided that $\left\|X^{k} f\right\|_{L^{p}(\mathbb{G})} \neq 0$.

For weights $w, v$ satisfying (3.2) we have 
Theorem 4.3. Let $1 \leq p \leq q<\infty$ satisfy (3.2). Suppose that $v$ is a weight and $w$ is a doubling weight such that

$$
\left\|f-f_{B}\right\|_{L_{w}^{q}(B)} \leq C_{0} w(B)^{1 / q} v^{\prime}(B)^{1 / p^{\prime}} \rho(B)^{-Q+1}\|X f\|_{L_{v}^{p}(B)}
$$

for all balls $B$ and $f \in \Lambda^{1}(\mathbb{G})$ where $v^{\prime}=v^{-1 /(p-1)}\left(v^{\prime}(B)^{1 / p^{\prime}}=\operatorname{esssup}_{x \in B} v^{-1}(x)\right.$ when $p=1)$. Then

$$
\begin{aligned}
\left\|X^{k} f\right\|_{L_{w}^{q}(B)} \leq & C w(B)^{1 / q} \rho(B)^{-Q-k}\|f\|_{L^{1}(B)} \\
& +C w(B)^{1 / q} v^{\prime}(B)^{1 / p^{\prime}} \rho(B)^{-Q+1}\left\|X^{k+1} f\right\|_{L_{v}^{p}(B)}
\end{aligned}
$$

for all balls $B$ and $f \in \Lambda^{k+1}(\mathbb{G})$ where $C$ is independent of $f$.

As corollaries, we have the following containment relation of function spaces: Corollary 4.4. Let $1 \leq p \leq q<\infty$ and let $\Omega, W, v$ and $w$ be as in Theorem 3.7 such that

$$
\left\|f-f_{B}\right\|_{L_{w}^{q}(B)} \leq A\|X f\|_{L_{v}^{p}(B)}
$$

for all $B \in W$ and $f \in \Lambda^{1}(\Omega)$. Then $E_{v, k+1}^{p}(\Omega) \subset E_{w, k}^{q}(\Omega)$ for all $k \in \mathbb{N}$.

Corollary 4.5. Let $\Omega$ and $W$ be as in Theorem 3.7. Suppose $1 \leq p \leq q<\infty$ and $w$ a doubling weight such that

$$
\left\|f-f_{B}\right\|_{L_{w}^{p}(B)} \leq A\|X f\|_{L_{w}^{p}(B)}
$$

for all $B \in W$ and $f \in \Lambda^{1}(\Omega)$. Then $f \in E_{w, k}^{p}(\Omega)$ if and only if $f \in L_{w, k}^{p}(\Omega)$.

Theorem 4.6 (Local). Let $1 \leq p \leq q<\infty, v \in A_{p}$ and $w$ a doubling weight such that (4.1) holds. Let $i, k \in \mathbb{N}, 1 \leq i<k$. Then

$$
\begin{aligned}
\left\|X^{i} f\right\|_{L_{w}^{q}(B)} \leq & C w(B)^{1 / q} \rho(B)^{-i} v(B)^{-1 / p}\|f\|_{L_{v}^{p}(B)} \\
& +C w(B)^{1 / q} v(B)^{-1 / p} \rho(B)^{k-i}\left\|X^{k} f\right\|_{L_{v}^{p}(B)}
\end{aligned}
$$

for all $f \in \Lambda^{k}(B)$.

Theorem 4.7 (Global). Let $1 \leq p \leq q<\infty, v \in A_{p}$ and $w$ a doubling weight such that (4.1) holds. Let $i, k \in \mathbb{N}, 1 \leq i<k$ and $-i<\lambda<k-i$. Then

$$
\left\|X^{i} f\right\|_{L_{w}^{q}(\mathbb{G})} \leq C\|f\|_{L_{v}^{p}(\mathbb{G})}^{1-(\lambda+i) /(k)}\left\|X^{k} f\right\|_{L_{v}^{p}(\mathbb{G})}^{(\lambda+i) /(k)}
$$

for all $f \in \Lambda^{k}(\mathbb{G})$ and $\left\|X^{k} f\right\|_{L_{v}^{q}(\mathbb{G})} \neq 0$ if and only if

$$
\rho(B)^{\lambda} w(B)^{1 / q} \leq C v(B)^{1 / p}
$$

for all balls $B$. 
We remark that the global theorems (3.1), (3.3), (3.4), and (4.2), and the sufficient part of theorem (4.7) follow from the local results, i.e., theorems (3.5), (3.2), (4.1) and (4.6). In order to get the global results from the local ones, we need to patch the local inequalities together by using a covering lemma concerning balls of equal sizes and then optimize the radius of balls to minimize some quantities obtained from the local inequalities. Details can be found in [L3].

\section{Poincaré inequalities with higher order derivatives}

In this section we give some Poincaré type inequalities with higher order derivatives on the right hand side of the inequalities. Poincaré inequalities of first orders for general Hömander's vector fields were first established by Jerison for $p=q$ and improved in [L1], and sharp nonweighted inequalities were first proved in [L2] for $p>1$. The case for $p=1$ was established in [FLW] including sharp weighted inequalities for all $p \geq 1$. There have been extensive study and numerous papers on how one weaker Poincaré inequality implies other strong one. We shall not address this issue here.

The case discussed here is just a special case of general Hörmander vector fields. We recall that $P_{k}(f, B)$ is a ploynomial on $\mathbb{G}$ of degree $\leq k-1$ such that

$$
\int_{B} X^{i}\left(f-P_{k}(f, B)\right)=0, \text { for all } 0 \leq i<k,
$$

and $\pi_{k}(B) f$ is the projection polynomial with degree less than $k$ of $f$ onto $B$, whose existence is guaranteed by the projection theorems (2.4) and (2.6). We recall that

$$
\operatorname{esssup}_{x \in B}\left|\pi_{k}(B) f(x)\right| \leq C \rho(B)^{-Q}\|f\|_{L^{1}(B)}
$$

with $C$ independent of $f$ and $B$. Moreover, $\pi_{k}(B)$ is linear and $\pi_{k}(B) P=P$ for all $P \in \mathcal{P}_{k}$, the collection of polynomials on $\mathbb{G}$ of degree less than $k$. We recall that $Q$ is the homogeneous dimension of $\mathbb{G}$.

Theorem 5.1. Suppose that $B \subset \mathbb{G}$ is any metric ball and $f \in \Lambda^{k}(\bar{B})$. Then we have for any $0 \leq j<i \leq k$

$$
\begin{aligned}
\left(\frac{1}{|B|} \int_{B} \mid X^{j}(f(x)-\right. & \left.\left.P_{k}(f, B)(x)\right)\left.\right|^{q_{i j}} d x\right)^{\frac{1}{q_{i j}}} \\
& \leq C \rho(B)^{i-j}\left(\frac{1}{|B|} \int_{B}\left|X^{i}\left(f(x)-P_{k}(f, B)(x)\right)\right|^{p} d x\right)^{\frac{1}{p}}
\end{aligned}
$$

for all $1 \leq p<\frac{Q}{i-j}$ and $q_{i j} \leq \frac{p Q}{Q-(i-j) p}$, where $C$ is independent of $B$ and $f$.

As a simple corollary of this theorem we get the perhaps most commonly used Poincaré inequality below. This follows from theorem (5.1) by noting that $X^{k} P_{k}(f, B)=0$ because $P_{k}(f, B)$ is a polynomial of degree less than $k$. 
Corollary 5.2. Suppose that $B \subset \mathbb{G}$ is any metric ball and $f \in \Lambda^{k}(\bar{B})$. Then we have

$$
\left(\frac{1}{|B|} \int_{B}\left|f(x)-P_{k}(f, B)(x)\right|^{\frac{Q p}{Q-k p}} d x\right)^{\frac{Q-k p}{p Q}} \leq C \rho(B)^{k}\left(\frac{1}{|B|} \int_{B}\left|X^{k} f(x)\right|^{p} d x\right)^{\frac{1}{p}}
$$

for all $1 \leq p<\frac{Q}{k}$, where $C$ is independent of $B$ and $f$.

The proof of theorem (5.1) follows from the repeated use of the standard Poincaré inequality. In the proof we have used the property of the approximation polynomial $P_{k}(f, B)$, i.e., the vanishing integral properties (see theorem $(2.5)$.

The above theorems hold as well when we replace the approximation polynomial $P_{k}(f, B)$ by the projection polynomial $\pi_{k}(B) f$. We can actually get more refined (somehow different) version for this polynomial by dropping the polynomial on the right hand side. Thus we can state the above type theorems into one.

Theorem 5.3. Suppose that $B \subset \mathbb{G}$ is any metric ball and $f \in \Lambda^{k}(\bar{B})$. Then we have for any $0 \leq j<i \leq k$

$$
\left(\frac{1}{|B|} \int_{B}\left|X^{j}\left(f(x)-\pi_{k}(B) f(x)\right)\right|^{q_{i j}} d x\right)^{\frac{1}{q_{i j}}} \leq C \rho(B)^{i-j}\left(\frac{1}{|B|} \int_{B}\left|X^{i} f(x)\right|^{p} d x\right)^{\frac{1}{p}}
$$

for all $1 \leq p<\frac{Q}{i-j}$ and $q_{i j} \leq \frac{p Q}{Q-(i-j) p}$, where $C$ is independent of $B$ and $f$.

Since we do not necessarily have the vanishing integral property for the projection polynomial $\pi_{k}(B) f$, the proof of theorem (5.3) does not follow immediatly. Indeed, the proof relies on the approximation polynomilas in an elegant way.

Next we will give similar Poincaré inequalities on domains satisfying the socalled chain condition as defined in (1.1).

Theorem 5.4. Let $\Omega \in \mathcal{F}(\sigma, N)$ be a Boman chain domain in $\mathbb{G}$ and $f \in \Lambda^{k}(\Omega)$. Then we have

$$
\left(\int_{\Omega}\left|f(x)-P_{k} f(x)\right|^{\frac{Q p}{Q-k p}} d x\right)^{\frac{Q-k p}{p Q}} \leq C\left(\int_{\Omega}\left|X^{k} f(x)\right|^{p} d x\right)^{\frac{1}{p}}
$$

for all $1 \leq p<\frac{Q}{k}$, where $C$ is independent $f$ and $\Omega$, but only on the Boman chain constants $\sigma$ and $N$, and $P_{k} f$ can be taken either as $P_{k}\left(f, B_{0}\right)$ or $\pi_{k}\left(B_{0}\right) f$ for the central ball $B_{0}$.

The proof of theorem (5.4) follows from the Corollary (5.2) and Theorem (5.3).

We remark here that more general forms of the above theorem can be given for $1 \leq j<i \leq k$ as in Theorems (5.1). But we shall not give here. 


\section{References}

[B] B. Bojarski, Remarks on Sobolev imbedding inequalities, Complex Analysis, Lecture Notes in Math., 1351, Springer-Verlag, 1989, 52-68.

[BKL] S. Buckley, P. Koskela and G. Lu, Boman equals John, XVIth Rolf Nevanlinna Colloquium, Eds. Laine/Martio, Walter de Gruyter \&Co., 92-99.

[CKN1] L. Caffarelli, R. Kohn and L. Nirenberg, First order interpolation inequalities with weights, Compositio Math. 53 (1984), 259-275.

[CKN2] _ Partial regularity of suitable weak solutions of the Navier-Stokes equations, Comm. Pure Appl. Math. 35 (1982), 771-831.

[CW] S. Chanillo and R. L. Wheeden, Weighted Poincaré and Sobolev inequalities and estimates for the Peano maximal functions, Amer. J. Math. 107 (1985), 1191-1226.

[CoW] R. R. Coifman and G. Weiss, Analyse harmonique non-commutative sur certains espaces homogènes, Springer Lecture Notes in Mathematics , 242, 1971.

[C] S. K. Chua, Weighted Sobolev interpolation inequalities, Proc. Amer. Math. Soc. 117 (1993), 449-457.

[Ca] A. Calderon, Inequalities for the maximal function relative to a metric, Studia Math. 57 (1976), 297-306.

[F] G.B. Folland, Subelliptic estimates and function spaces on nilpotent Lie groups, Ark. Mat. 13 (1975), 161-207.

[FS1] G. B. Folland and E. M. Stein, Estimates for the $\bar{\partial}_{b}$-complex and analysis on the Heisenberg group, Comm. Pure Appl. Math. 27 (1974), 429-522.

[FS2] _ Hardy spaces on homogeneous groups, Princeton U. Press, 1980.

[FGW] B. Franchi, C. Gutierrez and R. L. Wheeden, Weighted Sobolev-Poincaré inequalities for Grushin type operators, Comm. Partial Diff. Eq. 19 (1994), 523-604.

[FLW] B. Franchi, G. Lu and R. L. Wheeden, Representation formulas and weighted Poincaré inequalities for Hormander vector fields, Inst. Fourier (Grenoble) 45 (1995), 577-604.

[GN] N. Garofalo and D. Nhieu, Isoperimetric and Sobolev inequalities for CarnotCarathedory spaces and the existence of minimal surfaces, Comm. Pure Appl. Math. 49 (1996), 1081-1144.

[GW1] C. E. Gutierrez and R. L. Wheeden, Sobolev interpolation inequalities with weights, Trans. Amer. Math. Soc. 323 (1991), 263-281.

[GW2] _ Harnack's inequalities for degenerate parabolic equations, Comm. Partial Diff. Eq. 16 (1991), 4-5, 745-770.

[GW3] _ Mean value and Harnack inequalities for degenerate parabolic equations, Colloq. Math. 60/61 (1990), 157-194.

[H] L. Hörmander, Hypoelliptic second order differential equations, Acta Math. 119 (1967), 147-171.

[J] D. Jerison, The Poincaré inequality for vector fields satisfying Hörmander's condition, Duke Math. J. 53 (1986), 503-523.

[L1] G. Lu, Weighted Poincaré and Sobolev inequalities for vector fields satisfying Hörmander's condition and applications, Revista Mat. Iberoamericana 8 (1992), 367-439.

[L2] - The sharp Poincaré inequality for free vector fields: an endpoint result, Revista Mat. Iberoamericana 10 (1994), 453-466.

[L3] _ Polynomials on stratified groups and Sobolev interpolation inequalities on nonisotropic Folland-Stein spaces, Preprint, (1997).

[L4] _ A Fefferman-Phong type inequality for degenerate vector fields and applications, PanAmerican Mathematical J., 6 (1996), 37-57.

[L5] _ Fundamental solution and $L^{p}$ estimates for subelliptic Schrödinger operators on stratified groups, Preprint, (1995).

[Lin] C. S. Lin, Interpolation inequalities with weights, Comm. Partial Diff. Eq. 11 (1986), 1515-1538. 
[M] J. Moser, On a pointwise estimate for parabolic differential equations, Comm. Pure. Appl. Math. 24 (1971), 727-740.

[RS] L. Rothschild and E. M. Stein, Hypoelliptic differential operators and nilpotent groups, Acta Math. 137 (1976), 247-320.

[ST] J. Stromberg and A. Torchinsky, Weighted Hardy spaces, Lecture Notes in Mathematics, No. 1381, Springer, New York 1989.

[SW] E. Sawyer and R. L. Wheeden, Weighted inequalities for fractional integrals on Euclidean and homogeneous spaces, Amer. J. Math. 114 (1992), 813-874.

Department of Mathematics, Wright State University, Dayton, OH 45435

E-mail address: gzlu@math.wright.edu 